\title{
The burden and dynamics of hospital-acquired SARS-CoV-2 in England
}

Ben Cooper ( $\square$ ben.cooper@ndm.ox.ac.uk)

University of Oxford https://orcid.org/0000-0002-9445-7217

\section{Stephanie Evans}

PHE/UKHSa

\section{Yalda Jafari}

LSHTM

\section{Thi Mui Pham}

Utrecht University

Cherry Lim

University of Oxford

Mark Pritchard

University of Oxford

\section{Diane Pople}

Public Health England/UKHSA

\section{Victoria Hall}

Public Health England/UKHSA

\section{James Stimson}

Public Health England/UKHSA https://orcid.org/0000-0003-1855-6229

\section{David Eyre}

University of Oxford https://orcid.org/0000-0001-5095-6367

Jonathan Read

Lancaster University

Christl Donnelly

University of Oxford

\section{Peter Horby}

University of Oxford

\section{Conall Watson}

University of Oxford

\section{Sebastian Funk}

London School of Hygiene \& Tropical Medicine https://orcid.org/0000-0002-2842-3406 Julie Robotham

Public Health England/UKHSA

\section{Gwen Knight}


LSHTM

Mo Yin

University of Oxford

\section{Biological Sciences - Article}

Keywords: SARS-CoV-2, hospital-based transmission, England

Posted Date: November 30th, 2021

DOI: https://doi.org/10.21203/rs.3.rs-1098214/v1

License: (a) (i) This work is licensed under a Creative Commons Attribution 4.0 International License. Read Full License 


\section{The burden and dynamics of hospital-acquired SARS-CoV-2 in}

\section{England}

4 Authors: Ben S Cooper ${ }^{1,2}$, Stephanie Evans ${ }^{3}$, Yalda Jafari ${ }^{4}$, Thi Mui Pham ${ }^{5}$, Mo Yin ${ }^{1,2,6,7}$, Cherry

5 Lim $^{1,2}$, Mark G Pritchard ${ }^{1}$, Diane Pople ${ }^{3}$, Victoria Hall ${ }^{3}$, James Stimson ${ }^{3}$, David W Eyre ${ }^{8,9,10,11,}$

6 Jonathan M Read ${ }^{12}$, Christl A Donnelly ${ }^{13,14}$, Peter Horby ${ }^{1}$, Conall Watson ${ }^{1}$, Sebastian Funk ${ }^{4}$, Julie

$7 \quad$ V Robotham $^{3,11 *}$, Gwenan M Knight ${ }^{*}$

8

9 Affiliations:

1 Oxford Centre for Global Health Research, Nuffield Department of Medicine,

University of Oxford, Oxford, United Kingdom

2 Mahidol-Oxford Tropical Medicine Research Unit, Faculty of Tropical Medicine,

Mahidol University, Thailand

3 Healthcare Associated Infections and Antimicrobial Resistance Division, National

Infection Service, PHE, Colindale, London, UK

4 Centre for mathematical modelling of infectious diseases, IDE, EPH, London School of Hygiene \& Tropical Medicine, London, UK

6 Division of Infectious Disease, Department of Medicine, National University Hospital,

Singapore

5 Julius Center for Health Sciences and Primary Care, University Medical Center Utrecht, Utrecht University, Utrecht, The Netherlands

Department of Medicine, National University of Singapore 
8 Big Data Institute, Nuffield Department of Population Health, University of Oxford, Oxford, United Kingdom

9 Oxford University Hospitals, NHS Foundation Trust, Oxford, United Kingdom

10 NIHR Oxford Biomedical Research Centre, University of Oxford, Oxford, United

11 NIHR Health Protection Research Unit in Healthcare Associated Infections and Antimicrobial Resistance at University of Oxford in partnership with Public Health England, Oxford, United Kingdom

12 Lancaster Medical School, Lancaster University, Lancaster, UK

Department of Statistics, University of Oxford, Oxford, United Kingdom MRC Centre for Global Infectious Disease Analysis, Department of Infectious Disease Epidemiology, Imperial College London

${ }^{*}$ These authors contributed equally to this work 
Abstract

Hospital-based transmission played a dominant role in MERS-CoV and SARS-CoV

3 epidemics but large-scale studies of its role in the SARS-CoV-2 pandemic are lacking. Such

4 transmission risks spreading the virus to the most vulnerable individuals and can have wider-

5 scale impacts through hospital-community interactions. Using data from acute hospitals in

6 England we quantify within-hospital transmission, evaluate likely pathways of spread and

7 factors associated with heightened transmission risk, and explore the wider dynamical

8 consequences. We show that hospital transmission is likely to have been a major contributor

9 to the burden of COVID-19 in England. We estimate that between June 2020 and March 2021

0 between 95,000 and 167,000 patients acquired SARS-CoV-2 in hospitals with nosocomially-

1 infected patients likely to have been the main sources of transmission to other patients.

2 Increased transmission to patients was associated with hospitals having fewer single rooms

3 and lower heated volume per bed. Moreover, we show that reducing hospital transmission

4 could substantially enhance the efficiency of punctuated lockdown measures in suppressing community transmission. These findings reveal the previously unrecognised scale of hospital transmission, have direct implications for targeting of hospital control measures, and highlight the need to design hospitals better-equipped to limit the transmission of future high consequence pathogens. 
6 Hospital transmission played a central role in the spread of Middle East respiratory syndrome 7 coronavirus (MERS-CoV) and severe acute respiratory syndrome coronavirus (SARS-CoV) in 8 human populations ${ }^{1,2}$, and multiple reports have indicated that SARS-CoV-2 is capable of 9 spreading efficiently in healthcare settings ${ }^{3-11}$ and is associated with poor outcomes ${ }^{12,13}$. However, 0 attempts to fully document the extent of hospital transmission using systematically-collected

1 national data or to take a data-driven approach to quantifying the drivers and dynamical

2 consequences of such transmission are lacking. Addressing these knowledge gaps is important:

3 hospital transmission directly affects patients likely to have multiple factors associated with poor 4 outcomes; it puts healthcare workers (HCWs) at risk and compromises their ability to provide safe 5 patient care; it disrupts service delivery; and it can play a major role in disseminating infection to 6 vulnerable groups in the community. Moreover, because non-pharmaceutical interventions in the

7 community do not affect rates of transmission from infected patients and HCWs in hospitals,

8 hospital transmission can have important effects on epidemic dynamics during lockdown periods.

9 Understanding such transmission has implications for both ongoing epidemics and for threats from 0 new variants in highly vaccinated populations.

2 We use data from 145 National Health Service (NHS) acute hospital trusts (organisational units 3 containing one or more acute care hospitals), excluding only those caring exclusively for children.

4 These trusts contained 356 hospitals, had a combined bed capacity of approximately 100,000, (over $598 \%$ of the total NHS general and acute care bed capacity in England in 2020) and employed 6859,134 full-time equivalent HCWs, 2.5\% of the working-age population of England. From 20th 7 March 2020, all such trusts completed a daily situation report which included essential information 8 on the prevalence and incidence of SARS-CoV-2 infection, the number of patients admitted with 
9 SARS-CoV-2 infection and of staff absences due to COVID-19. From 5th June 2020, a

0 classification of the likely source of infection based on ECDC criteria was also required ${ }^{14}$. This

1 was determined by the interval between hospital admission and date of onset of PCR-confirmed

2 infection in hospitalised patients: community onset infections were defined as those with an interval

3 of two days or fewer; an interval of 3-7 days led to a classification of indeterminate healthcare-

4 associated; those with an interval of 8-14 days were classified as probable healthcare-associated;

5 and intervals of 15 days or more were classified as definite healthcare-associated. Since few

6 patients have hospital stays exceeding seven days and many nosocomially-infected patients will be

7 discharged before testing positive, such definitions necessarily capture only a proportion of

8 hospital-acquired infections.

9

0 We make use of these data, linked with other national data sets to infer the number of hospital-

1 acquired infections in England between June 2020 and February 2021, the pathways of nosocomial

2 transmission, factors potentially modulating such transmission, and to understand its dynamical

3 consequences.

7 Results

9 Between 10th June 2020 and 17th February 2021 a total of 16,950 and 19,355 SARS-CoV-2

0 infections in hospital inpatients met the criteria for definite and probable healthcare-associated

1 infections respectively. To estimate the total number of hospital-acquired infections we multiply the

2 recorded number of definite healthcare-associated infections by the reciprocal of the proportion of

3 hospital-acquired infections that we expect to meet these "definite healthcare-associated" criteria. 
4 Using the empirical length-of-stay distribution, the estimated incubation period distribution, and the 5 profile of PCR test sensitivity as a function of time since infection ${ }^{15}$ (Fig. 1 a-c) we estimate that a 6 policy of PCR testing symptomatic patients would detect $26 \%(90 \%$ credible interval $(21 \%, 30 \%))$ 7 of hospital-acquired infections, with $12 \%(10 \%, 14 \%)$ of all such infections meeting criteria for 8 definite healthcare-associated infection (Fig. 1 d-f). Adding asymptomatic PCR testing on days of 9 stay 3 and 6 (as recommended by national screening guidance in England) increases the proportion 0 detected to $33 \%(26 \%, 38 \%)$ but does not substantively alter the proportion classified as definite 1 healthcare-associated. Augmenting symptomatic PCR tests with testing for all patients at seven-day 2 intervals (a policy adopted by some hospitals in England) increases the proportion of hospital3 acquired infections detected to $44 \%(39 \%, 47 \%)$, and the proportion classified as definite 4 healthcare-associated to $17 \%(16 \%, 18 \%)$. These low probabilities for detection and classification 5 as definite healthcare associated are a consequence of the typically short lengths of patient stay and 6 low PCR sensitivities early in the course of infection (Fig. 1 b-c).

8 Combining these estimates with the number of reported definite healthcare-associated infections,

9 we infer the number of hospital-acquired infections under two sets of assumptions. First, we assume 0 patient testing followed national guidance which specifies testing of symptomatic patients but 1 includes no asymptomatic testing after day seven post-admission. This provides a plausible lower 2 bound for the chance of identifying hospital-acquired infections and thus an upper bound for the 3 estimated numbers of such infections. Second, we assume testing for all patients at seven-day 4 intervals post-admission in addition to symptomatic testing of patients. This provides a plausible 5 upper bound for the chance of identifying hospital-acquired infections and thus a lower bound on 6 the estimated numbers of such infections. Using definite healthcare-associated infections only, this 7 yielded as an upper bound a mean $(90 \% \mathrm{CrI})$ estimate for the number of nosocomial infections of $8143,000(123,000,167,000)$ and a lower bound of $99,000(95,000,104,000)$. During this period 
9 there were 9.2 million hospital admissions from 5.0 million individual patients, so we estimate that 0 between $1 \%$ and $2 \%$ of admissions resulted in a hospital-acquired SARS-CoV-2 infection. Similar 1 estimates were obtained in sensitivity analyses using more granular length-of-stay data, while 2 repeating the analysis using probable and definite healthcare-associated infections yielded estimates 3 that were 20-30\% higher (Supplementary Information: Section 2.1).

4 5 6 7 8 9 
a

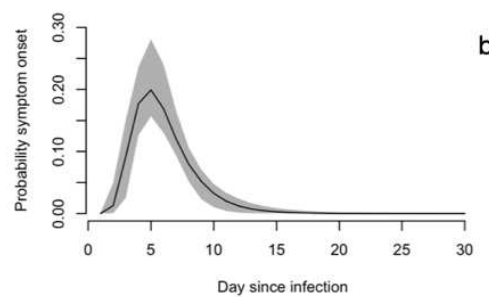

d
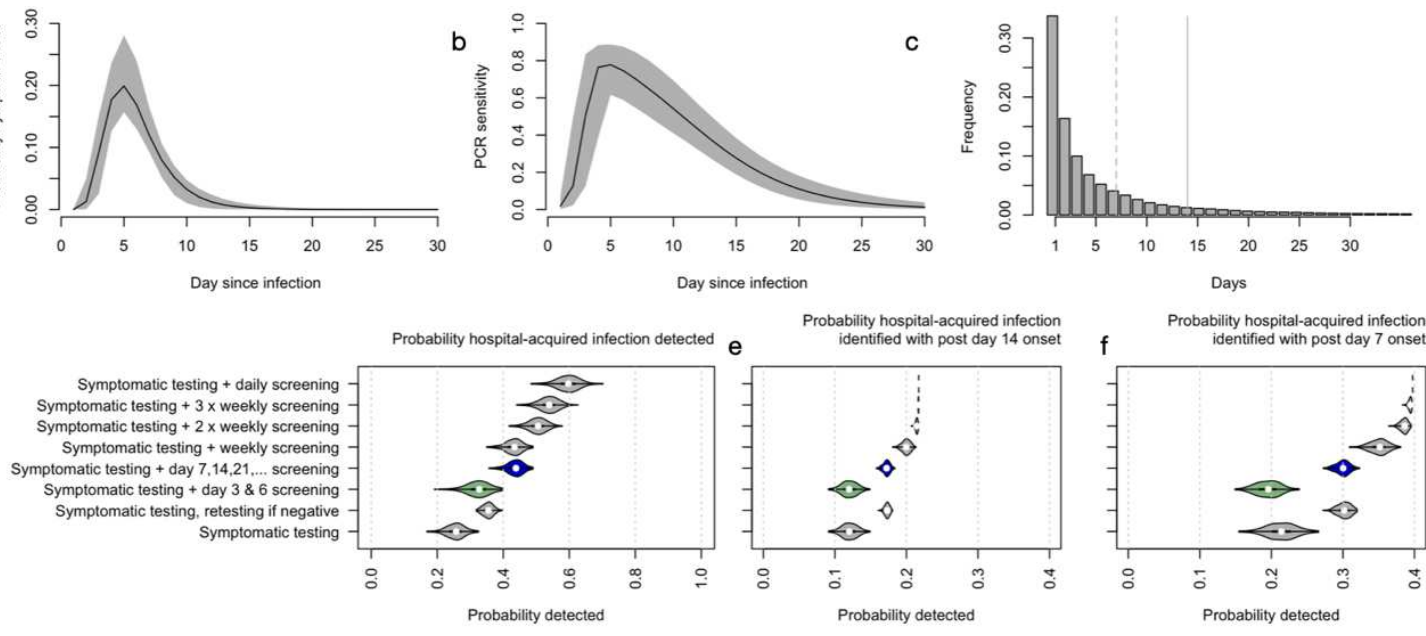

g

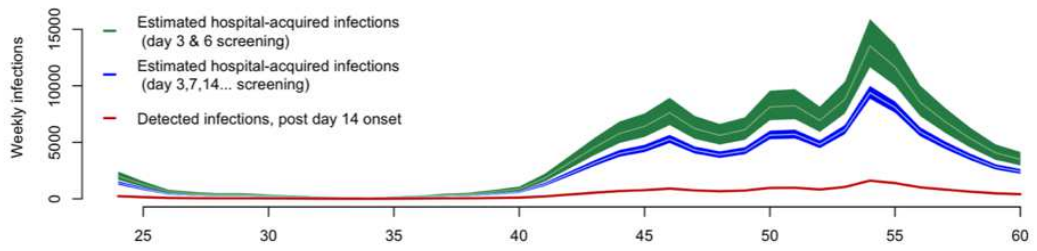

Week number

Fig. 1 | Quantifying the probability of observing hospital-acquired infections and estimating the total number of such

infections. Model inputs are shown in the top row and include the incubation period distribution (a) ${ }^{16}$, the PCR sensitivity profile (b) ${ }^{15}$, and the length-of-stay distribution (c) for patients who were not admitted with COVID-19. In (c) the minimum lengths of stays needed to be classified as a probable or definite healthcare-associated infection are shown by dashed and solid vertical lines.

Estimates of the probabilities that patients with hospital-acquired SARS-CoV-2 infections have a PCR positive test while in hospital under different screening policies (d), and estimates of the probabilities that they both screen positive and meet the post-14 day onset criteria to be considered a "definite" healthcare-associated infection (e) or the post-7 day criteria to be classified as a probable or definite healthcare-associated infection (f) are shown in the middle row, with the Public Health England screening recommendations highlighted in green and the policy of screening all patients at seven day intervals after admission is highlighted in blue (note that in contrast to this policy, weekly and 2 and $3 \mathrm{x}$ weekly policies screen on fixed days of the week). The bottom panel (g) shows the estimated total number of hospital-acquired infections across adult NHS trusts in England assuming the screening policies highlighted in the middle row based on recorded "definite healthcare-associated infections".

To quantify drivers of transmission to patients and HCWs we linked these data to national data-sets

1 (Fig. 2 e-1) capturing information on hospital characteristics potentially affecting transmission,

$2 \mathrm{HCW}$ vaccination, and regional prevalence of the Alpha variant. As no direct measurements of 
hospital ventilation were available, we used hospital building heated volume per bed as a proxy.

4 This analysis was restricted to 96 of the 145 trusts for which complete data were available and used

5 negative binomial auto-regression models where the dependent variable was either the weekly

6 number of patients with healthcare-associated infections or the imputed weekly number of $\mathrm{HCW}$

7 infections. Independent variables were selected based on biological plausibility. Mechanistic

8 considerations informed the parameterisation of the dispersion terms and the inclusion of additive

9 effects for exposures to community-acquired patient infections, hospital-acquired patient infections,

0 and infected HCWs (Fig. 2, top row), combined with multiplicative effects of trust characteristics

1 (Fig. 2, middle row), HCW vaccine coverage and Alpha variant prevalence (Fig. 2, bottom row).

3 Amongst the additive terms the strongest predictor of new healthcare-associated infections was the

4 number of patients in the same trust with healthcare-associated infections the previous week (Fig.

53 3); thus one patient with a newly identified healthcare-associated infection the previous week was

6 associated with an additional $1.07(95 \% \mathrm{CrI} 0.93,1.19)$ hospital-acquired infections in patients the

7 following week (setting variables representing hospital characteristics to their mean values, and in

8 the absence of the Alpha variant or vaccine effects). Additive effects associated with patient

9 exposures to infected HCWs and patients admitted with SARS-CoV-2 were smaller, though the

0 larger number of such exposures increased their contribution to patient infections (Fig. 3F).

2 Considering multiplicative effects associated with trust characteristics, increased availability of

3 single rooms was associated with reduced incidence of healthcare-associated infections in patients

4 with an incidence rate ratio (IRR) for a one SD increase in single room availability (corresponding

5 to a $15 \%$ increase in the percentage of beds as single rooms) of $0.91(0.87,0.97)$, while heated

6 volume per bed was associated with a similar reduction (IRR $0.90(0.84,0.97)$ for a one SD

7 increase corresponding to an increase per bed of $207 \mathrm{~m}^{3}$, and older hospital buildings were also 
8 associated with reduced hospital transmission (Fig. 3). These effects were not seen for infections in

9 HCWs. HCW vaccination was associated with substantial reduction in transmission to patients

0 linked to exposures to infected HCWs, and large reductions in the overall rate of infection in

1 HCWs. Increased Alpha variant prevalence was associated with large increases in the rates of

2 infection in both patients and HCWs.

4 Negative controls can help assess the likelihood that associations between exposures and outcomes

5 in observational studies result from relationships which are not directly causal ${ }^{17}$. We used as a

6 negative outcome control the number of patients admitted with community-acquired SARS-CoV-2

7 infection. Assuming most hospital admissions with SARS-CoV-2 result from community

8 transmission, this outcome would not be expected to have a strong association with hospital

9 characteristics and exposures. If associations between hospital exposures and this control outcome

0 are similar to those for hospital-acquired infections, it would suggest that confounding is a plausible

1 explanation. Note, however, that since some SARS-CoV-2 admissions from the community will

2 result from the readmission of patients infected in hospital some link is expected. In all models

3 considered with this control outcome, there was no strong association with the number of

4 healthcare-associated infections or with the single room provision, strengthening the evidence that

5 these both play a causal role in the incidence of hospital-acquired infections (Supplementary

6 Information: Tables S15-S17). However, both heated volume per bed and HCW vaccination

7 coverage showed similar negative associations with the control outcome as reported for healthcare-

8 associated infection outcomes, indicating the need for caution when considering whether these

9 reported associations might reflect direct causal effects.

1 To help interpret estimated regression coefficients we performed a series of simulation studies,

2 generating synthetic transmission data-sets from a multitype branching process model, applying an 
3 observation model to obtain partially observed infection data, and replicating the above analysis.

4 This analysis indicated that when the outcome was patient hospital-acquired infections, regression

5 coefficients typically underestimated the expected number of secondary cases per case when only a

6 proportion of hospital-acquired infections were observed, though represented good approximations

7 as the proportion approached 1 (Extended Data Fig. 4).

8 


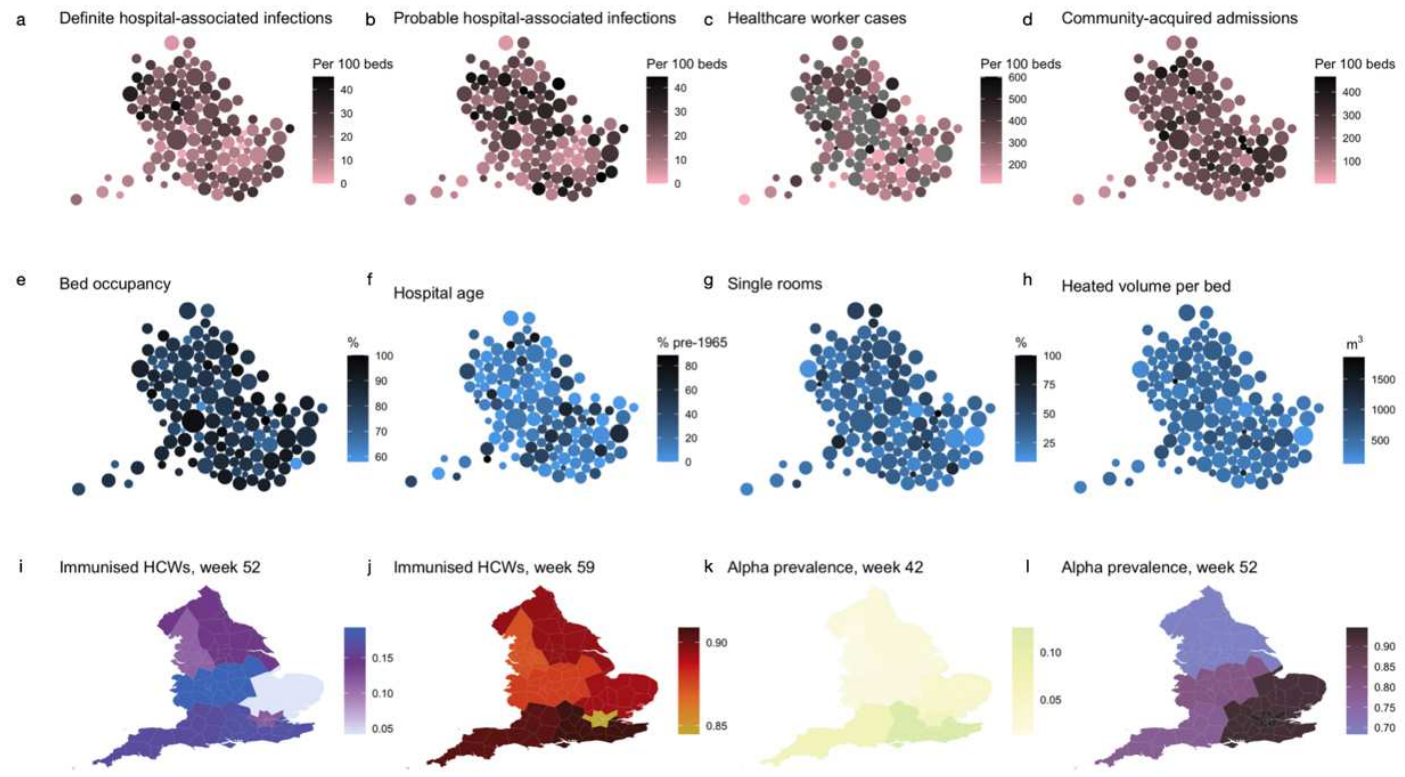

2 Fig. 2 | Summary of data used in the analysis. First row: data from situation reports related to SARS-CoV-2

3 infection in England showing variation between trusts. Each circle corresponds to one NHS trust scaled by the number

4 of available beds. Shading indicates cumulative totals to the end of the period considered (February 172021 ).

5 Geographic locations are approximate. Cumulative number of hospital-associated infections in patients per 100 hospital

6 beds with first positive sample $>14$ days after admission (a); hospital-associated infections in patients with first positive

7 sample $>7$ days after admission (b); imputed cumulative number of cases in healthcare workers (c) with grey shading

8 indicating missing data; infections in hospitalised patients with community onset (d). Second row: trust-level data

9 characteristics from the third quarter of 2020: bed occupancy (e); age of acute hospital buildings in the trust expressed

0 as a weighted average of the percentage of hospital buildings constructed in 1964 or earlier, where weights are the

1 hospital gross internal floor areas (f); number of single room beds per trust (including isolation rooms) as a percentage

2 of the number of general and acute beds available in the last quarter of $2020(\mathbf{g})$; heated volume per bed (h). Third row:

3 a snapshot of regional HCW immunisation data at two time points showing the proportion of HCWs who had received

4 at least one vaccine dose at least three weeks earlier $(\mathbf{i}, \mathbf{j})$, and regional data on the proportion of confirmed infections

5 due to the Alpha variant (in both cases voronoi tessellations centred on the location of the largest hospital in each trust

6 are shown). 


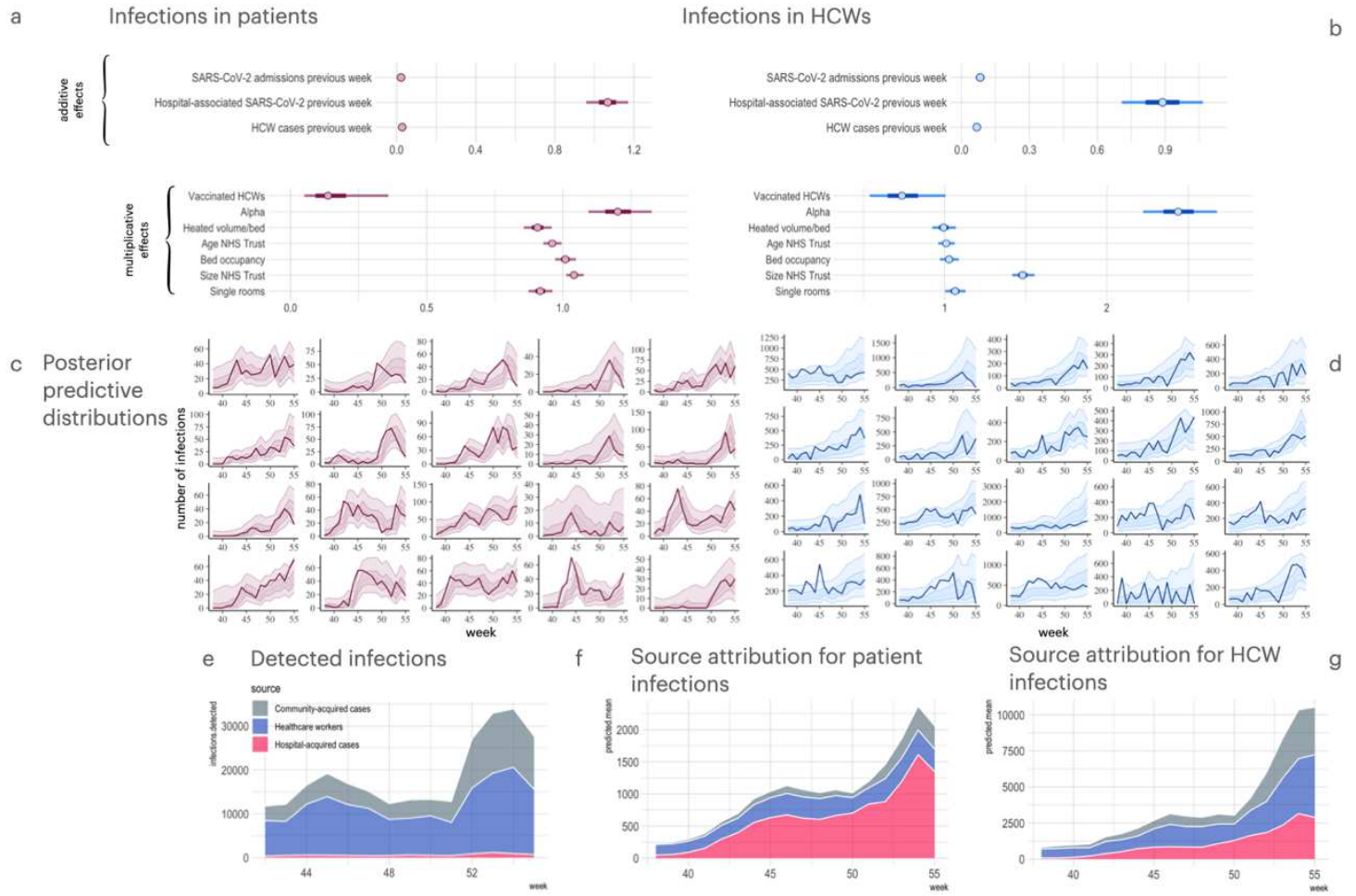

3 Fig. 3 | Factors associated with nosocomial SARS-CoV-2 acquisitions in patients HCWs and predictive

4 distributions. a, $\mathbf{b}$ show additive effects associated with categories of host infections and multiplicative effects of vaccine coverage in healthcare workers, Alpha prevalence and trust characteristics (posterior means, $50 \%$ and $90 \%$ CrIs are shown). For multiplicative effects, values below one indicate an association with reduced infection rates. Note that in the model for infections in patients (a) HCW vaccine coverage acts by modulating transmission associated with

8 infected healthcare workers, while in the model for infections in healthcare workers it has a global effect, modulating

9 the overall rate of infection. Associated posterior predictive distributions for the number of infections by week in the 20

$0 \quad$ largest trusts are shown (c and $\mathbf{d}$; solid line corresponds to observed values and shaded regions correspond to 50\% and

$190 \%$ CrIs). The bottom row shows, for all trusts, classifications of detected infections (e) by week, and contributions to

2 predicted infections in patients (f) and HCWs (g) from the three categories of infected hosts predicted by the negative

3 binomial regression models. 
5 We used estimates from these analyses to inform a dynamic model coupling hospital and community dynamics. We considered three scenarios: high hospital transmission, corresponding to

7 self-sustaining within-hospital transmission; and intermediate and low hospital transmission, where

8 all hospital transmission rates were reduced by $25 \%$ and $50 \%$ respectively compared to the high

9 hospital transmission scenario (Figure 4). Community transmission rates were identical in all

0 scenarios.

1

2 While the level of hospital transmission had little overall impact on an unmitigated epidemic

3 (Extended Data Fig. 5), when community transmission is controlled through punctuated lockdowns,

4 the extent of hospital transmission can have a profound impact on overall epidemic dynamics. If

5 lockdowns are put in place for a fixed time period and then released in a stepwise manner (Fig. 4a-

6 i), the total infected population in the community decreases from $27 \%$ in the high hospital

7 transmission scenario to $12 \%$ and $7 \%$ in the intermediate and low transmission scenarios (Fig. 4g-i)

8 with corresponding increases of the percentages of HCWs infected from $21 \%$ to $52 \%$ and $91 \%$ (Fig.

9 4d-f). Conversely, if instigation and release of lockdowns is driven by threshold infection rates in

0 the community (Fig. $4 \mathrm{j}-\mathrm{u}$ ) the total number infected does not depend strongly on levels of hospital

1 transmission (Fig. 4j-o) but the time spent in lockdown and the efficiency with which lockdown

2 averts infections (Fig. $4 \mathrm{p}-\mathrm{u}$ ) is enhanced by reducing hospital transmission. These effects can be

3 substantial despite the fact that, at any one time, the number of patients and HCWs is less than $2 \%$

4 of the total population.

5 


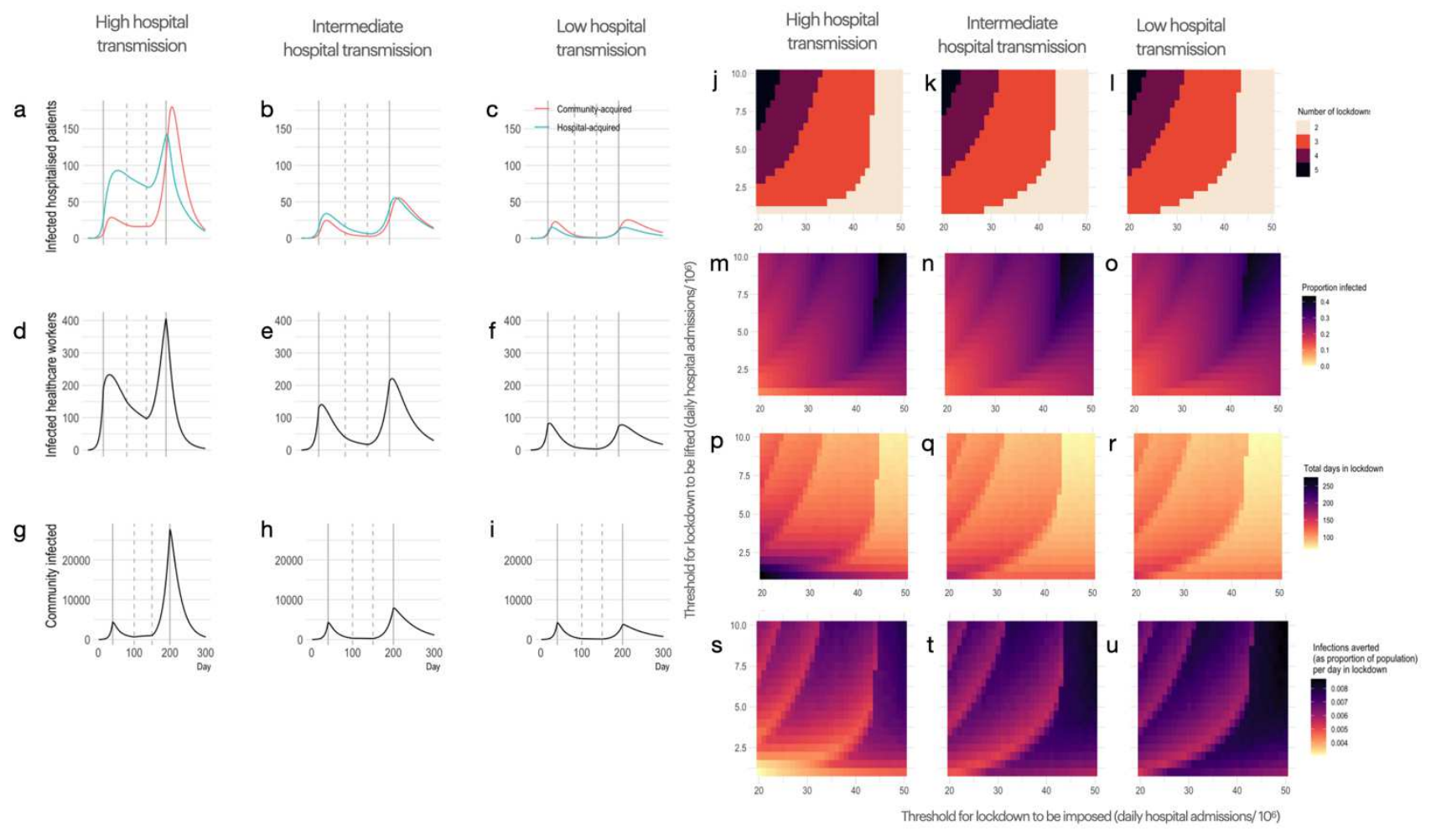

2 Fig. 4 | Dynamics of community and hospital infections. Results are shown from simulation runs under high (a,d,g),

3 intermediate $(\mathbf{b}, \mathbf{e}, \mathbf{h})$ and low $(\mathbf{c}, \mathbf{f}, \mathbf{i})$ rates of hospital transmission scenarios, where rates of hospital transmission in

4 intermediate and low scenarios are, respectively, 25\% and 50\% lower than the high hospital transmission scenario

5 without altering parameters related to community transmission. Assumed population sizes for community, hospital

6 inpatients and HCWs are 500,000, 1000, and 4000 respectively. Solid vertical lines correspond to initiation of

7 "lockdown" measures which are assumed to reduce person-to-person transmission rates in the community by $80 \%$ for

8 the first lockdown and 70\% for the second. The two broken vertical lines correspond to progressive release of lockdown

9 measures, here assumed to result in transmission rates in the community that are reduced by $70 \%$ (after 100 days) and

$040 \%$ (after a further 50 days) compared to the pre-intervention rate. The same three hospital transmission scenarios are

1 used when considering threshold-driven lockdown measures $(\mathbf{j}-\mathbf{u})$, when lockdown measures are initiated and released

2 based on per capita infection rates in the community being above or below pre-specified thresholds. In these scenarios,

3 when lockdown is in place person-to-person transmission rates in the community are assumed to be reduced by $90 \%$

4 compared with pre-intervention levels, while release of lockdown is followed by community transmission rates that are

$550 \%$ of those prior to the first lockdown. 
Discussion

0 Between $1 \%$ and $2 \%$ of hospital admissions are likely to have resulted in a hospital-acquired

1 SARS-CoV-2 infection during the "second wave" in England, with only a minority of these

2 infections correctly classified as "healthcare-associated". Time series data indicated that patients

3 who themselves acquired SARS-CoV-2 infection in hospital were the main drivers of transmission

4 to patients while transmission from both HCWs and nosocomially-infected patients were of similar

5 importance for transmission to HCWs. HCW vaccination was associated with large reductions in

6 infection rates and there was evidence that aspects of hospital building design could modulate such

7 transmission.

8 While lack of genomic data means we cannot conclusively demonstrate transmission, our findings

9 accord with focused local investigations with densely-sampled viral genome sequences. Such

0 studies indicate that many hospital-onset infections not meeting ECDC definitions for healthcare-

1 associated infection are hospital-acquired and highlight the importance of superspreading 5,18 . Such

2 superspreading is implicit in our negative binomial models which attribute $80 \%$ of detected patient-

3 patient transmission events from nosocomially-infected patients to $21 \%$ of infected patients. Also

4 aligned with our findings (Fig. $3 \mathbf{f}-\mathbf{g}$ ) are conclusions from local studies that hospital-acquired

5 infection in patients is primarily due to transmission from nosocomially infected patients, while

6 sources for HCW infections came from patients and HCWs in approximately equal proportions

$79,18,19$.

8 National infection prevention and control (IPC) guidance in England at the start of June 2020

9 emphasised respiratory and hand hygiene, use of face masks for patients and HCWs, cohorting of

0 patients and staff, environmental decontamination, ventilation, and staff social distancing. 
1 Screening of all patients for SARS-CoV-2 during the first seven days of their hospital stay was

2 recommended throughout the period, but some trusts went beyond these requirements by

3 performing weekly testing. Records of such measures were not kept at a national level and lack of

4 centrally collected data on trust-specific IPC measures means that effective interventions may have

5 gone unrecognised. Simulation studies, however, suggest that high-frequency asymptomatic

6 screening and rapid isolation of patients with suspected SARS-CoV-2 can substantially reduce

7 SARS-CoV-2 transmission in healthcare settings ${ }^{20,21}$ and highlight the importance of contact

8 tracing ${ }^{22}$.

9 The factors that make it hard to prevent SARS-CoV-2 transmission are relevant for hospitals

0 everywhere. While some well-resourced hospitals avoided large-scale nosocomial transmission in

1 early $2020^{23-25}$, even in high-income settings the extent of such transmission showed considerable

2 variation between hospitals ${ }^{8}$. Seroprevalence data prior to vaccination in HCWs also indicate a

3 high degree of heterogeneity between hospitals even within the same countries and are consistent

4 with high levels of nosocomial transmission in many settings (Extended Data Fig. S6). Hospitals in

5 resource-limited settings face particular challenges due to poorly-funded IPC activities, lack of

6 capacity to carry out routine testing, lack of isolation facilities, and high levels of patient crowding,

7 but attempts to systematically quantify the extent of such transmission outside high-income

8 countries are currently lacking.

0 Our findings have implications for control policies. First, they highlight the importance of early

1 identification and prompt initiation of control measures for patients with new hospital-acquired

2 infections and for other patients they may have infected. Second, they reinforce the need for

3 measures that reduce transmission from patients with asymptomatic infection in non-COVID

4 hospital areas, including improved ventilation, use of face coverings by patients and staff, increased

5 distancing between beds, minimising patient movements within and between wards, and promotion 
6 of hand hygiene ${ }^{26}$. Third, our findings support efforts to prioritise HCWs for COVID-19

7 vaccination both due to direct protection to $\mathrm{HCWs}$ and due to indirect protection offered to patients.

8 Fourth, the findings highlight the need to prioritise research into effective methods of reducing

9 hospital transmission of airborne pathogens, including ward design and air filtration systems ${ }^{27}$.

0 Finally, our findings show that hospital transmission can have a substantial impact on epidemic

1 dynamics. In particular, the role of hospital transmission in seeding COVID-19 into care homes and

2 other vulnerable groups in the community must be further investigated in light of the finding that

3 much of the hospital transmission is likely to be unobserved.

\section{References}

6 1. Cowling, B. J. et al. Preliminary epidemiological assessment of MERS-CoV outbreak in South $7 \quad$ Korea, May to June 2015. Euro Surveill. 20, 7-13 (2015).

8 2. Cooper, B. S. et al. Transmission of SARS in three Chinese hospitals. Trop. Med. Int. Health 14 Suppl 1, 71-78 (2009).

3. Meredith, L. W. et al. Rapid implementation of SARS-CoV-2 sequencing to investigate cases of health-care associated COVID-19: a prospective genomic surveillance study. Lancet Infect.

5. Lumley, S. F. et al. Epidemiological data and genome sequencing reveals that nosocomial transmission of SARS-CoV-2 is underestimated and mostly mediated by a small number of Dis. 20, 1263-1271 (2020).

4. Ellingford, J. M. et al. Genomic and healthcare dynamics of nosocomial SARS-CoV-2 transmission. eLife 10, (2021). highly infectious individuals. J. Infect. (2021).

6. Zhou, Q. et al. Nosocomial infections among patients with COVID-19, SARS and MERS: a rapid review and meta-analysis. Ann. Transl. Med. 8, 629 (2020). 
0 7. Richterman, A., Meyerowitz, E. A. \& Cevik, M. Hospital-Acquired SARS-CoV-2 Infection: Lessons for Public Health. JAMA 324, 2155-2156 (2020).

8. Read, J. M. et al. Hospital-acquired SARS-CoV-2 infection in the UK's first COVID-19

9. Mo, Y. et al. Transmission of community- and hospital-acquired SARS-CoV-2 in hospital settings in the UK: A cohort study. PLoS Med. 18, e1003816 (2021).

10. Shirreff, G. et al. How well does SARS-CoV-2 spread in hospitals? bioRxiv (2021) doi:10.1101/2021.09.28.21264066.

11. San, J. E. et al. Transmission dynamics of SARS-CoV-2 within-host diversity in two major hospital outbreaks in South Africa. Virus Evol 7, veab041 (2021).

12. Ponsford, M. J. et al. Burden of nosocomial COVID-19 in Wales: results from a multicentre retrospective observational study of 2508 hospitalised adults. Thorax (2021) doi:10.1136/thoraxjnl-2021-216964.

13. Hetemäki, I. et al. An outbreak caused by the SARS-CoV-2 Delta variant (B.1.617.2) in a secondary care hospital in Finland, May 2021. Euro Surveill. 26, (2021).

14. Surveillance definitions for COVID-19. https://www.ecdc.europa.eu/en/covid19/surveillance/surveillance-definitions.

15. Hellewell, J. et al. Estimating the effectiveness of routine asymptomatic PCR testing at different frequencies for the detection of SARS-CoV-2 infections. BMC Med. 19, 106 (2021).

16. Lauer, S. A. et al. The Incubation Period of Coronavirus Disease 2019 (COVID-19) From Publicly Reported Confirmed Cases: Estimation and Application. Ann. Intern. Med. 172, 577$582(2020)$

17. Lipsitch, M., Tchetgen, E. \& Cohen, T. Negative controls: a tool for detecting confounding and bias in observational studies. Epidemiology 21, 383-388 (2010).

18. Illingworth, C. et al. Superspreaders drive the largest outbreaks of hospital onset COVID-19 
infection. eLife (2021). doi:10.7554/elife.67308

6

19. Lindsey, B. B. et al. Characterising within-hospital SARS-CoV-2 transmission events: a retrospective analysis integrating epidemiological and viral genomic data from a UK tertiary care setting across two pandemic waves. medRxiv (2021) doi:10.1101/2021.07.15.21260537.

20. Chin, E. T. et al. Frequency of Routine Testing for Coronavirus Disease 2019 (COVID-19) in High-risk Healthcare Environments to Reduce Outbreaks. Clin. Infect. Dis. (2020) doi:10.1093/cid/ciaa1383.

21. Evans, S., Agnew, E., Vynnycky, E. \& Robotham, J. The impact of testing and infection prevention and control strategies on within-hospital transmission dynamics of COVID-19 in English hospitals. Philos. Trans. Royal. Soc. B. (2021) doi:10.1098/rstb.2020.0268

22. Pham, T. M. et al. Interventions to control nosocomial transmission of SARS-CoV-2: a modelling study. Preprint at https://www.medrxiv.org/content/10.1101/2021.02.26.21252327v2 (2021).

23. Baker, M. A. et al. Low risk of COVID-19 among patients exposed to infected healthcare workers. Clin. Infect. Dis. (2020) doi:10.1093/cid/ciaa1269.

24. Nguyen, L. H. et al. Risk of COVID-19 among front-line health-care workers and the general community: a prospective cohort study. Lancet Public Health 5, e475-e483 (2020).

25. Sikkema, R. S. et al. COVID-19 in health-care workers in three hospitals in the south of the Netherlands: a cross-sectional study. Lancet Infect. Dis. 20, 1273-1280 (2020).

26. Cheng, Y. et al. Face masks effectively limit the probability of SARS-CoV-2 transmission. Science (2021) doi:10.1126/science.abg6296.

27. Mousavi, E. S., Kananizadeh, N., Martinello, R. A. \& Sherman, J. D. COVID-19 Outbreak and Hospital Air Quality: A Systematic Review of Evidence on Air Filtration and Recirculation. Environ. Sci. Technol. 55, 4134-4147 (2021).

28. Mizumoto, K., Kagaya, K., Zarebski, A. \& Chowell, G. Estimating the asymptomatic 
0 proportion of coronavirus disease 2019 (COVID-19) cases on board the Diamond Princess 1 cruise ship, Yokohama, Japan, 2020. Euro Surveill. 25, (2020).

2 29. Hall, V. J. et al. SARS-CoV-2 infection rates of antibody-positive compared with antibody3 negative health-care workers in England: a large, multicentre, prospective cohort study $4 \quad$ (SIREN). Lancet 397, 1459-1469 (2021).

5 30. Ferretti, L. et al. Quantifying SARS-CoV-2 transmission suggests epidemic control with digital $6 \quad$ contact tracing. Science 368, (2020). 


\section{Methods}

\section{Quantifying the number of hospital acquired infections}

0 To quantify the number of hospital-acquired infections in patients we first estimate the

1 probability, $\pi_{i j}$, that an infection acquired by a patient in trust $i$ in week $j$ is both detected by a PCR

2 test and meets the definition of a hospital-acquired infection. In the main analysis, this definition

3 requires the first positive specimen date to be 15 days or more after the day of hospital admission

4 and while the patient is in hospital. In general, $\pi_{i j}$ will depend on the testing policy in place at the

5 time, the sensitivity of the PCR test (which varies with time since infection), the length-of-stay

6 distribution in the trust for patients at risk of being infected with SARS-CoV-2 while in hospital,

7 the distribution of times from infection to onset of symptoms, and the proportion of infections

8 which are symptomatic. Consider an infection that a patient acquires $d$ days after the day the

9 patient is admitted to the hospital. The testing policy in place in the trust during the patient's stay,

0 the day of infection, and the incubation period distribution together determine the probability that a

1 patient is tested on day $k$ after the patient is infected (for $k=0,1,2,3 \ldots$ ). We assume the test has a

2 specificity of 1 . Let $\phi_{k}$ represent the probability of a positive PCR test result for a test taken $k$ days

3 after the date of infection, and let $\tau_{i j k}$ represent the probability that such a test is performed $k$ days

4 after the infection event. The probability that a patient admitted to trust $i$ in week $j$ with length of

5 stay $m$ infected on day of stay $d$ (where $d \leq m$ ) has a positive PCR test while in hospital is given

6 by $P_{i j m d}=1-\prod_{k=d \ldots m}\left(1-\tau_{i j(k-d)} \phi_{k-d}\right)$. 
8 For such a hospital-acquired infection to meet the definition of a confirmed "definite" hospital-

9 acquired infection requires the first positive sample to be taken 15 or more days after the day the

0 patient is admitted to the trust. The probability, $P^{\prime}{ }_{i j m d}$, of this happening for patients with length of

1 stay $m$ infected on day $d$ is clearly zero for $m<15$, otherwise it is given by the probability that

2 there is no positive test before day 15 and at least one positive test after. For $d \geq 15$ this probability

3 is identical to $P_{i j m d}$, otherwise it is given by

$4 \quad P_{i j m d}^{\prime}=\prod_{k=d \ldots 14}\left(1-\tau_{i j(k-d)} \phi_{k-d}\right)\left(1-\prod_{k=15 \ldots m}\left(1-\tau_{i j(k-d)} \phi_{k-d}\right)\right)$.

5 The probability that a new nosocomial infection in trust $i$ and week $j$ is detected is then given by

$6 \pi_{i j}=\sum_{m, d} \gamma_{i j m d} P_{i j m d}$ while the probability that a new nosocomial infection in trustiand week $j$ is

7 both detected and meets the definition of a confirmed nosocomial infection is

$8 \pi_{i j}=\sum_{m, d} \gamma_{i j m d} P_{i j m d}^{\prime}$, where $\gamma_{j i m d}$ is the probability that, given a new hospital-acquired

9 infection in trust $i$ in week $j$ occurs, it occurs in a patient with length of stay $m$ on day of stay $d$. If

$0 \quad \lambda_{\text {im }}$ represents the probability that a patient at risk of nosocomial infection with SARS-CoV-2

1 admitted to trust $i$ has a length of stay of $m$ days, then, on a given day, the expected proportion of

2 patients who both have a length of stay of $m$ days and are currently on day of staydis given by

$3 \quad \psi_{\text {imd }}=\lambda_{i m} m /\left(d \sum_{n} \lambda_{i n} n\right)$ if $m \geq d$ and 0 otherwise. Analysis of individual-level patient data

4 indicates that while daily risk of infection changes over calendar time, it does not vary appreciably

5 with day of stay $d$ for typical lengths of stays ${ }^{9}$, and we therefore approximate $\gamma_{i j m d}$ (the

6 conditional probability of a new infection occurring on day of stay $d$ in a patient with length of stay

$7 \quad m$ given that an infection occurs) by $\psi_{\text {imd }}$ which we estimate based on the reported lengths of stays

8 of completed patients episodes of patients admitted to each trust over the time period considered.

9 Given these estimates of $\pi_{i j}$ and the observed number of confirmed hospital-acquired infections,

$0 y_{i j}$, we estimated the the total number of hospital-acquired infections (combining observed and

1 unobserved), $z_{i j}$, by applying Bayes' formula: $P\left(z_{i j} \mid y_{i j}, \pi_{i j}\right)=P\left(y_{i j} \mid z_{i j}, \pi_{i j}\right) P\left(z_{i j}\right) / P\left(y_{i j} \mid \pi_{i j}\right)$ 
2 where $P\left(y_{i j} \mid z_{i j}, \pi_{i j}\right)$ represents the binomial likelihood of observing $y_{i j}$ infections, $P\left(z_{i j}\right)$ is the

3 prior distribution for the total number of infections, which we take to be uniform, and we calculate

$4 \quad P\left(y_{i j} \mid \pi_{i j}\right)$ using the formula $P\left(y_{i j} \mid \pi_{i j}\right)=\sum_{l} P\left(y_{i j} \mid \pi_{i j}, z_{i j}=l\right) P\left(z_{i j}=l\right)$.

5 We consider a number of different testing policies, which determine the $\tau_{i j k}$ values. The minimal

6 testing policy, which involves the fewest tests, requires only that patients displaying symptoms of

7 COVID-19 are tested, and we assume all such patients are tested on a single occasion. When this

8 policy is in place the times of testing of patients with hospital-acquired infections, in relation to the

9 time of infection, is determined by the incubation period and such a test is assumed to be performed

0 if and only if the patient develops symptoms on or before the day of discharge. A second testing

1 policy extends this by assuming that in the event of a negative screening result from a patient with

2 symptoms, daily testing will continue to be performed until patient discharge, the first positive test,

3 or three consecutive negative tests (whichever occurs first). In these analyses we use reported

4 estimates of sensitivity of the PCR test as a function of time since infection ${ }^{15}$. We consider

5 additional testing policies which combine symptomatic testing with routine asymptomatic testing.

6 In these policies all patients who have not already tested positive are screened at predetermined

7 intervals using the same PCR test. In these policies we consider weekly, twice weekly, three times

8 weekly and daily testing of all in-patients as well as a policy of testing twice in the first week of

9 stay (in accordance with national guidance in England). For a given length-of-stay distribution,

0 incubation period distribution, PCR sensitivity profile, and probability that infection is symptomatic

1 the calculations outlined above to determine the probability that an infection is detected or both

2 detected and identified as a hospital-acquired infection are deterministic, and require no simulation.

3 We account for uncertainty in these quantities through a Monte Carlo sampling scheme, at each

4 iteration sampling new values for PCR sensitivities, the incubation period distribution and the

5 proportion of infections which are symptomatic. For PCR sensitivities, we directly sample from the

6 posterior distribution reported by Hellewell et al ${ }^{15}$. For the incubation period we assume a 
7 lognormal distribution, and sample the parameters for these from normal distributions with means 8 (SDs) of $1.621(0.064)$ and $0.418(0.069)$ as estimated by Lauer et al ${ }^{16}$. Estimates of the proportion 9 of infections which are symptomatic are taken from Mizumoto et al ${ }^{28}$ and this quantity is sampled 0 from a normal distribution with mean (SD) of $0.82(0.012)$. Length-of-stay distributions are directly 1 obtained from SUS for NHS acute trusts excluding: i) patients who were admitted with PCR-

2 confirmed COVID-19, ii) patients who had samples taken in the first seven days of their hospital 3 stay which were PCR positive for SARS-CoV-2; and iii) patients with a length-of-stay of less than 4 one day. In the primary analysis we use aggregate length-of-stay data for all trusts taken from the 512 month period from March 1st 2020. We also present results from two sensitivity analyses: in the

6 first we use trust-specific $\lambda_{\text {im }}$ values; in the second we allow for the possibility that length-of-stay 7 distributions change over time and use period-specific empirical length-of-stay distributions from 8 the four three month periods: March-May 2020, June-August 2020, September-November 2020, 9 and December 2020 - February 2021.

1 Quantifying drivers of nosocomial transmission

3 We used generalised linear mixed models to quantify factors associated with nosocomial

4 transmission. In these models the dependent variable was either the observed number of confirmed 5 hospital-acquired infections in trust $i$ and week $j$ amongst patients, $y_{i j}$, or the imputed number of 6 infections in healthcare workers, $y_{i j}^{\prime}$. Three classes of independent variables were considered: i)

7 known exposures to others in the same trust infected with SARS-CoV-2 to account for within-trust 8 temporal dependencies, with separate terms corresponding to exposures in the previous week to 9 patients with community-onset SARS-CoV-2 infections $\left(z_{i(j-1)}\right)$, patients with hospital-acquired

0 SARS-CoV-2 $\left(y_{i(j-1)}\right)$, and healthcare workers with SARS-CoV-2 $\left(y_{i(j-1)}^{\prime}\right)$; ii) characteristics of 1 the trusts which were considered, a priori, to be plausibly linked to hospital transmission: bed 
2 occupancy, provision of single rooms, age of hospital buildings, heated air volume per bed, and size

3 (number of acute care beds); iii) regional data including vaccine coverage amongst healthcare

4 workers and the proportion of isolates represented by the alpha variant. Models were formulated to

5 reflect presumed mechanisms generating the data, and we used negative binomial models with

6 identity link functions allowing the number of exposures to different categories of SARS-CoV-2

7 infections to contribute additively to the predicted number of weekly detected infections, while

8 allowing for multiplicative effects of the other terms. In models where the dependent variable

9 represented hospital-acquired infections in patients, the healthcare worker vaccination effect was

0 assumed to act only through a multiplicative term affecting transmission related to exposures to

1 healthcare workers. In contrast, when the dependent variable represented infections in healthcare

2 workers, vaccine exposure was allowed to have a multiplicative effect on the overall expected

3 number of infections. Formally, we define the full model for infections in patients in trust $i$ and

4 week $j$ (which we refer to as model P1.1.1) as:

5

$6 y_{i j} \sim \operatorname{neg} \operatorname{bin}\left(\mu_{i j}, \varphi_{i j}\right)$, where $\mu_{i j}$ represents the mean and the variance is given by $\mu_{i j}+\mu_{i j}^{2} / \varphi_{i j}$.

$7 \quad$ In the full model $\mu_{i j}=\left(a_{i}+b y_{i(j-1)}+c_{i j} y_{i(j-1)}^{\prime}+d z_{i(j-1)}\right) m_{i j} n_{i j}$

$$
m_{i j}=\exp \left(q \times \text { singlerooms }_{i}+r \times \text { trustsize }_{i}+s \times \text { occupancy }_{i(j-1)}+t \times \text { trustage }_{i j}\right.
$$

$$
\left.+u \times \text { trustvolumeperbed }_{i j}\right)
$$

$$
n_{i j}=\exp \left(w \times \text { proportionalphavariant }_{i j}\right)
$$

$$
c_{i j}=c \times \exp \left(v \times h c w v a x_{i(j-1)}\right)
$$

$$
\varphi_{i j}=\varphi_{0}+k_{i} y_{i(j-1)}
$$

$$
a_{i} \sim N\left(a_{0}, \sigma_{a}^{2}\right)
$$

$4 \quad k_{i} \sim N\left(k_{0}, \sigma_{k}^{2}\right)$. 
6 The expression for the dispersion parameter of the negative binomial distribution, $\varphi_{i j}$, reflects the

7 fact that the sum of $n$ independent negative binomially distributed random variables with mean $\mu$

8 and dispersion parameter $\varphi$ will itself have a negative binomial distribution with mean $n \mu$ and

9 dispersion parameter $n \varphi$. Thus, in the idealised case that each of $n$ nosocomially infected patients

0 in one week has a fully observed negative binomially distributed offspring distribution the next

1 week with mean $\mu$ and dispersion parameter $\varphi$, then the total number of nosocomial infections

2 observed would have a negative binomial distribution with parameters $n \mu$ and $n \varphi$. The $a_{i}$

3 represents a trust level random effect term to account for within-trust dependency. We also

4 considered two nested models, P1.1.0 and P1.0.0 obtained by setting the terms $q, r, s, t$ and $u$ to 0

5 in both cases (i.e. removing the trust-level terms) and by additionally setting the terms $v$ and $w$ to

6 zero in the latter case (i.e. removing regional vaccine and variant related terms).

8 A similar model was used when the dependent variable was healthcare worker infections, except

9 that the healthcare worker vaccine effect was included in the multiplicative term $m_{i j}$ instead of

0 operating only through the $c_{i j}$ term.

2 We used normal $(0,1)$ prior distributions by default for model parameters, except for variance terms $3 \sigma_{a}^{2}$ and $\sigma_{k}^{2}$ for which we used half-Cauchy $(0,1)$ prior distributions, and $\varphi$ for which a half-

$4 \operatorname{normal}(0,1)$ prior distribution was specified for the transformed parameter $1 / \sqrt{\varphi_{0}}$. All analysis was

5 performed in Stan using the rstan package version 2.21.1 in R (Stan Development Team (2020).

6 "RStan: the R interface to Stan." R package version 2.21. 2, http://mc-stan.org/) running each

7 model with four chains using 1000 iterations for warmup and 5000 iterations for sampling. 
9 In the main analysis, we used weekly aggregated data, counting week numbers as one plus the

0 number of complete seven day periods since January 1 st 2020 . We included only acute hospital

1 trusts in this analysis, and excluded trusts which predominantly admitted children.

3 Independent variable data

5 Vaccine coverage in HCWs by week and NHS region were taken from the SIREN study ${ }^{29}$, and we

6 classified healthcare workers as being immunised if they had received at least one vaccine dose 3 or

7 more weeks previously. Otherwise they were considered un-immunised. Trust-specific data used in

8 the analysis not related to infections (number of single rooms, size, age, heated volume and bed

9 occupancy) were derived from the Estates Returns Information Collection from NHS Digital

0 (https://digital.nhs.uk/data-and-information/publications/statistical/estates-returns-information-

1 collection) including only the following site types: general acute hospital, community hospital (with

2 inpatient beds), mixed service hospital, specialist hospital (acute only). The number of single rooms

3 was expressed as the number of beds in single rooms in the trust (including single bedrooms for

4 patients with and without en-suite facilities and isolation rooms) divided by the number of general

5 and acute beds reported as being available in the trust in the last quarter of 2020. Hospital size was

6 taken as the number of hospital beds available in the trust. A hospital building age score was taken

7 as a weighted average of the proportion of floor area across hospital sites that was built before

8 1965, where weights were taken as the building floor area. SARS-CoV-2 variant data consisted of

9 the proportion of characterised isolates that were attributed to the Alpha variant in each week for

0 each NHS region. The prevalence of the Alpha variant by region and over time was determined by

1 S-gene status from PCR tests provided by Public Health England

2 (https://github.com/epiforecasts/covid19.sgene.utla.rt). 
Imputation method for weekly number of infections in HCWs

7 Situation reports included fields allowing quantification of nosocomial transmission and number of 8 HCWs isolated due to COVID-19 from June 5th 2020, but analysis here is restricted to data from 9 week 42 (beginning 14th October 2020) to week 55 (beginning 13th January 2021) reflecting the 0 date range from which all fields used in the analysis were consistently reported. Because situation 1 reports did not explicitly include data on the number of infections in HCWs, only the number of 2 HCWs absent due to COVID-19 on each day, we imputed the weekly number of infections amongst 3 HCWs at each trust. We did this by first subtracting from the number of reported HCW COVID-19 4 absences in each trust on each day the reported number of such absences due to contact tracing and 5 isolation policies (reflecting likely COVID-19 exposures in the community). Then, assuming that 6 each HCW with COVID-19 was isolated for 10 days and assuming that durations of these absences 7 were initially uniformly distributed (starting from week 36) the number imputed to have entered 8 isolation on day $t$ was taken to be equal to the the number of COVID-19 absences on day $t+1$

9 minus the number of such absences on day $t$ plus the number imputed to have entered isolation ten 0 days early. For each trust we performed these calculations ten times, sampling the initial duration of 1 staff absences from a multinomial distribution assigning equal probabilities to durations of $1 \ldots 10$ 2 days, and then took the average (rounded to the nearest integer) of these samples. In some trusts it 3 was evident that some days with missing $\mathrm{HCW}$ isolation data had been coded as zeroes. When such 4 zeroes fell between daily counts in excess of 10 we treated them as missing data and replaced them 5 with the last number carried forward. Any negative numbers for daily imputed HCW infections 6 resulting from the above procedure were replaced with zeroes. While data on healthcare-associated 7 infections in patients was recorded consistently by all trusts throughout the inclusion period, in 8 some trusts data on HCW absences due to COVID-19 were missing or had been recorded 
9 inconsistently throughout the inclusion period. Excluding such trusts and those with missing data

0 for independent variables left 96 out of the original 145 trusts included in the analysis.

\section{Negative control outcomes}

4 We used as a negative outcome control the number of patients admitted with confirmed community-acquired SARS-CoV-2 infection as the outcome variable. We performed three analyses

6 where we adopted this negative control as our dependent variable, corresponding to model P1.1.1, $7 \quad$ P1.1.0, and P1.0.0 as defined above.

\section{Hospital-community interaction model}

1 We modelled hospital-community interaction using ordinary differential equations for an expanded

2 susceptible/exposed/infectious/removed (SEIR) model. This model included separate

3 compartments for people in the community, patients in hospital and HCWs (Figure S1). We used

4 the model to explore the impact of hospital transmission on overall epidemic dynamics with the aim

5 of providing qualitative insights. We compared outcomes from high, medium and low hospital

6 transmission scenarios where the primary epidemic control measure was restricting rates of contact

7 in the community ("lockdowns") which was assumed to have no direct impact on contact rates

8 within hospitals. Full model details and parameters are given below. In the interest of simplicity,

9 we neglect hospitalisation of HCWs who account for approximately $1 \%$ of the total population.

2 a) For the hospitalised population 
$4 \alpha S_{C}$

$$
\frac{\mathrm{d} S_{H}}{\mathrm{~d} t}=\frac{-\beta_{H, H} S_{H}\left(I 1_{H}+I 2_{H}\right)-\beta_{H^{\prime}, H} S_{H} I_{H}^{\prime}-\beta_{H C W, H} S_{H} I 1_{H C W}}{N_{H}}-\mu S_{H}+
$$

${ }^{5} \alpha S_{C}$

$$
\frac{\mathrm{d} S_{H}}{\mathrm{~d} t}=\frac{-\beta_{H, H} S_{H}\left(I 1_{H}+I 2_{H}\right)-\beta_{H^{\prime}, H} S_{H} I_{H}^{\prime}-\beta_{H C W, H} S_{H} I 1_{H C W}}{N_{H}}-\mu S_{H}+
$$

6

$$
\frac{\mathrm{d} E 1_{H}}{\mathrm{~d} t}=\frac{\beta_{H, H} S_{H}\left(I 1_{H}+I 2_{H}\right)+\beta_{H^{\prime}, H} S_{H} I_{H}^{\prime}+\beta_{H C W, H} S_{H} I 1_{H C W}}{N_{H}}-\gamma_{1} E 1_{H}-
$$

$7 \mu E 1_{H}+\alpha E 1_{C}$

8

$9 \quad \frac{\mathrm{d} E 2_{H}}{\mathrm{~d} t}=\gamma_{1} E 1_{H}-\gamma_{2} E 2_{H}-\mu E 2_{H}+\alpha E 2_{C}$

0

$1 \quad \frac{\mathrm{d} I 1_{H}}{\mathrm{~d} t}=\gamma_{2} E 2_{H}-\rho_{1} I 1_{H}-\mu I 1_{H}+\alpha I 1_{C}$

2

$3 \quad \frac{\mathrm{d} I 2_{H}}{\mathrm{~d} t}=\rho_{1} I 1_{H}-\rho_{2} I 1_{H}-\mu I 2_{H}+\alpha I 2_{C}$

4

$5 \quad \frac{\mathrm{d} I_{H}^{\prime}}{\mathrm{d} t}=\pi_{H} \rho_{2} I 2_{H}-\rho_{3} I_{H}^{\prime}-\mu^{\prime} I_{H}^{\prime}+\alpha^{\prime} I_{C}^{\prime}$

6

$7 \quad \frac{\mathrm{d} R_{H}}{\mathrm{~d} t}=\left(1-\pi_{H}\right) \rho_{2} I 2_{H}+\rho_{3} I_{H}^{\prime}-\mu R_{H}+\alpha R_{C}$

8

9

0

1 b) For the community population

2

$3 \alpha S_{C}$

$$
\frac{\mathrm{d} S_{C}}{\mathrm{~d} t}=\frac{-\phi \beta_{C, C} S_{C}\left(I 1_{C}+I 2_{C}\right)-\phi \beta_{C, C}^{\prime} S_{C} I_{C}^{\prime}-\beta_{H C W, C} S_{C} I 1_{H C W}}{N_{C}}+\mu S_{H^{-}}
$$


$\begin{aligned} \frac{\mathrm{d} E 1_{C}}{\mathrm{~d} t} & =\frac{\phi \beta_{C, C} S_{C}\left(I 1_{C}+I 2_{C}\right)+\phi \beta_{C, C}^{\prime} S_{C} I_{C}^{\prime}+\beta_{H C W, C} S_{C} I 1_{H C W}}{N_{C}}-\gamma_{1} E 1_{C}+ \\ \mu E 1_{H}-\alpha E 1_{C} & \end{aligned}$

5

$6 \frac{\mathrm{d} E 2_{C}}{\mathrm{~d} t}=\gamma_{1} E 1_{C}-\gamma_{2} E 2_{C}+\mu E 2_{H}-\alpha E 2_{C}$

$7 \frac{\mathrm{d} I 1_{C}}{\mathrm{~d} t}=\gamma_{2} E 2_{C}-\rho_{1} I 1_{C}+\mu I 1_{H}-\alpha I 1_{C}$

8

$9 \frac{\mathrm{d} I 2_{C}}{\mathrm{~d} t}=\rho_{1} I 1_{C}-\rho_{2} I 2_{C}+\mu I 2_{H}-\alpha I 2_{C}$

$0 \quad \frac{\mathrm{d} I_{C}^{\prime}}{\mathrm{d} t}=\pi_{C} \rho_{2} I 2_{C}-\rho_{3} I_{C}^{\prime}+\mu^{\prime} I_{H}^{\prime}-\alpha^{\prime} I_{C}^{\prime}$

$1 \frac{\mathrm{d} R_{C}}{\mathrm{~d} t}=\left(1-\pi_{C}\right) \rho_{2} I 2_{C}+\rho_{3} I_{C}^{\prime}+\mu R_{H}-\alpha R_{C}$

2

3

4 c) For HCWs

5

$6 \frac{\mathrm{d} S_{H C W}}{\mathrm{~d} t}=\frac{-\beta_{H, H C W} S_{H C W}\left(I 1_{H}+I 2_{H}\right)-\beta_{H, H C W}^{\prime} S_{H C W} I_{H}^{\prime}-\beta_{H C W, H C W} S_{H C W}\left(I 1_{H C W}+I 2_{H C W}\right)-\phi \beta_{C, H C W} S_{H C W}\left(I 1_{C}+I 2_{C}\right)}{N_{H C W}}$

7

$\frac{\mathrm{d} E 1_{H C W}}{\mathrm{~d} t}=\frac{\beta_{H, H C W} S_{H C W}\left(I 1_{H}+I 2_{H}\right)+\beta_{H, H C W}^{\prime} S_{H C W} I_{H}^{\prime}+\beta_{H C W, H C W} S_{H C W}\left(I 1_{H C W}+I 2_{H C W}\right)+\phi \beta_{C, H C W} S_{H C W}\left(I 1_{C}+I 2_{C}\right)}{N_{H C W}}-$

9

$0 \quad \frac{\mathrm{d} E 2_{H C W}}{\mathrm{~d} t}=\gamma_{1} E 1_{H C W}-\gamma_{2} E 2_{H C W}$

$1 \frac{\mathrm{d} I 1_{H C W}}{\mathrm{~d} t}=\gamma_{2} E 2_{H C W}-\rho_{1} I 1_{H C W}$

2

$3 \frac{\mathrm{d} I 2_{H C W}}{\mathrm{~d} t}=\rho_{1} I 1_{H C W}-\rho_{2} I 2_{H C W}$ 


$$
\begin{aligned}
& { }_{4} \frac{\mathrm{d} I_{H C W}^{\prime}}{\mathrm{d} t}=\pi_{H C W} \rho_{2} I 2_{H C W}-\rho_{3} I_{H C W}^{\prime} \\
& 5 \frac{\mathrm{d} R_{H C W}}{\mathrm{~d} t}=\left(1-\pi_{H C W}\right) \rho_{2} I 2_{H C W}+\rho_{3} I_{H C W}^{\prime}
\end{aligned}
$$

\section{Data availability}

8 The data that support the findings of this study are available as described below. All COVID-19

9 data for NHS trusts are publicly available either by direct download from

0 https://www.england.nhs.uk/statistics/statistical-work-areas/covid-19-hospital-activity/ or, for fields

1 related to numbers of healthcare-associated infections and length of stay, by submitting a Freedom

2 of Information request to NHS England: https://www.england.nhs.uk/contact-us/foi/. Variant data

3 are available from https://github.com/epiforecasts/covid19.sgene.utla.rt . Hospital building data are

4 available for direct download from the Estates Returns Information Collection from NHS Digital:

5 https://digital.nhs.uk/data-and-information/publications/statistical/estates-returns-information-

6 collection. Data relating to vaccine coverage in healthcare workers were collected as part of the

7 SIREN study (ISRCTN Number. ISRCTN11041050). Data from this study will be available

8 through the Health Data Research UK CO CONNECT platform and available for secondary

9 analysis once the SIREN study has completed reporting. Data and code to reconstruct the PCR

0 sensitivity profile are available from https://github.com/cmmid/pcr-profile. All analysis code for the

1 current paper is available from https://github.com/BenSCooper/nosocomial_COVID_England.

\section{Code availability}

$4 \quad$ All analysis code for the current paper is available from

5 https://github.com/BenSCooper/nosocomial_COVID_England. 
7 We are grateful to Susan Hopkins and the SIREN Study team for permission to use data on vaccination coverage of healthcare workers, with particular thanks to Sarah Foulkes and Edgar

9 Wellington who were instrumental in setting-up linkage between SIREN and National

0 Immunisation Management System (NIMS) records. We also thank Prof Catherine Noakes for

1 discussions. We acknowledge support from National Institute for Health Research and UK

2 Research and Innovation [COV0357; MR/V028456/1], National Institute for Health Research

3 Health Protection Research Unit (NIHR HPRU) in Healthcare Associated Infections and

4 Antimicrobial Resistance at Oxford University in partnership with Public Health England (PHE)

5 (NIHR200915), the NIHR Biomedical Research Centre, Oxford, and the NIHR HPRU in Emerging

6 and Zoonotic Infections at University of Liverpool in partnership with PHE, in collaboration with

7 Liverpool School of Tropical Medicine and the University of Oxford (NIHR200907). JMR

8 acknowledges support from the Medical Research Council (MR/V038613/1). BSC acknowledges

9 support from the Medical Research Council (MR/V028456/1). MY is supported by the Singapore

0 National Medical Research Council Research Fellowship (NMRC/Fellowship/0051/2017). CAD

1 acknowledges funding from the MRC Centre for Global Infectious Disease Analysis (reference

$2 \mathrm{MR} / \mathrm{R} 015600 / 1)$, jointly funded by the UK Medical Research Council (MRC) and the UK Foreign,

3 Commonwealth \& Development Office (FCDO), under the MRC/FCDO Concordat agreement and

4 is also part of the EDCTP2 programme supported by the European Union.

7 Author contributions

8 BSC, JVR, GMK, SE, TMP and DE conceptualized this work. BSC performed the statistical 9 analysis. BSC, SE and YJ developed the dynamic model. SE, YJ, CL, DP, VH, JS, SF, JVR, GMK 0 obtained, processed and verified the underlying data. BSC drafted the first version of the 
manuscript. All authors reviewed and edited subsequent versions of the manuscript. The

corresponding author attests that all listed authors meet authorship criteria and that no others meeting the criteria have been omitted. The corresponding author accepts full responsibility for the work and/or the conduct of the study, had access to the data, and controlled the decision to publish.

\section{Competing interest declaration}

7 The authors declare no competing financial interests. DWE declares personal fees from Gilead outside the submitted work.

Supplementary Information is available for this paper.

Correspondence should be addressed to ben.cooper@ndm.ox.ac.uk

Reprints and permissions information is available at www.nature.com/reprints

Extended data figure legends

Extended data Figure 1 | Flow diagram of the ordinary differential equation model.

Individuals are people in the community $(\mathrm{C})$, patients in hospital $(\mathrm{H})$ or healthcare workers $(\mathrm{HCW})$. Movements between the two exposed states (E1, E2), the two asymptomatic infectious states (I1, $\mathrm{I} 2$ ), and to the recovered state (R) are determined according to variables noted, in proportion to the 9 number of individuals in each category. A proportion $(\pi)$ of individuals in infectious state $\mathrm{I} 2$ have 0 severe symptomatic disease (I').

Extended data Figure $2 \mid$ Infections in patients. Posterior predictive distributions for all 96 trusts included in the analysis from model P1.1.1 where the outcome is probable and definite hospitalacquired infection. Details as in Figure 3.

Extended data Figure $3 \mid$ Infections in healthcare workers. Posterior predictive distributions for all 96 trusts included in the analysis from model P1.1.1 where the outcome is infections in HCWs.

Extended data Figure 4 | Results of a simulation study. Parameter estimates from fitting a negative binomial auto-regression model to simulated data under different probabilities for observing hospital-acquired infections in patients (a-f). The thick horizontal line indicates the component of the reproduction number used when simulating data (for example, in (a) each patient 2 with a hospital-acquired infection infects, on average, 0.6 other hospitalised patients). Red dots 
indicate the median from 100 simulations and the width in the violin plots is proportional to the density. Heatmaps (g-i) show how estimated model parameters from a negative binomial autoregression model (y-axis) map onto reproduction numbers (shown by the colour scale) for different proportions of hospital-acquired infections observed in patients (x-axis). Reproduction numbers correspond to expected numbers of secondary infections in patients from patients who themselves became infected in hospital (g), secondary infections in patients from healthcare workers $(\mathbf{h})$ and secondary infections in patients from patients admitted to hospital with COVID-19 (i).

Extended data Figure $5 \mid$ Additional output from deterministic mode. Dynamics of unmitigated epidemics unders scenarios of high, intermediate and low transmission in hospitals (a). Dynamics of epidemics under scenarios of high, intermediate and low transmission in hospitals when a single "lockdown" intervention is introduced on day 50, which has the effect of stopping $90 \%$ of community-based transmission but no effect on hospital-based transmission (b).

6 reported in the papers published before 16 May 2021. Dashed horizontal and vertical lines are the reported median values of seroprevalence in HCWs and in the community, respectively. The dots are coloured by the continent of which the survey population was in. The label for each dot shows country and survey period (i.e. 01/20 means January 2020). ). *The study from Iran surveyed 18 cities and classified the survey populations into high-risk populations (including HCWs, pharmacy employees, taxis drivers, cashiers of supermarket chains, and bank employees) and general populations in the same city over the same survey period. The bottom panel plot shows a zoomed in part of the top panel. 


\section{Supplementary Files}

This is a list of supplementary files associated with this preprint. Click to download.

- ManuscriptSI.docx

- Extendeddatafigure1.eps

- Extendeddatafigure2.eps

- Extendeddatafigure3.eps

- Extendeddatafigure4.eps

- Extendeddatafigure5.eps

- Extendeddatafigure6.eps 